\title{
Exuberant case of cutaneous metastasis of breast cancer ${ }^{*}$
}

\author{
Vítor Angelo Ferreira ${ }^{1}$ \\ Lucia Martins Diniz ${ }^{3,4}$
}

\author{
Karla Spelta ${ }^{2}$ \\ Elton Almeida Lucas ${ }^{1}$
}

DOI: http:/ /dx.doi.org/10.1590/abd1806-4841.20187157

\begin{abstract}
Cutaneous metastasis is the main cause of morbidity and mortality of cancer patients, denoting a guarded prognosis. The clinical spectrum of the disease is broad and can mimic benign conditions. The diagnosis depends on thorough clinical examination and complementary exams, with emphasis on the histopathological study and immunohistochemistry. Because it indicates a systemic relapse, it is necessary to intervene with intravenous chemotherapy, to which local therapies can be associated. We report the case of a 65-year-old woman with cutaneous metastasis from breast cancer treated 14 years back, manifested by extensive firm and infiltrated red-purple plaques on the sides of the trunk, with a course of six months. After evaluation, she was referred to the regional cancer center. She passed away one month and a half later.
\end{abstract}

Keywords: Breast neoplasms; Histology; Neoplasm metastasis; Recurrence

\section{INTRODUCTION}

Cutaneous metastasis is rare and represents a sign of malignancy and poor prognosis. It occurs in $0.7 \%$ to $10.4 \%$ of all cancers and represents $2 \%$ of cutaneous tumors. ${ }^{1}$

Breast cancer is the second most common cancer in women, with metastasis rates of up to $23.9 \%$, and is the first in causing cutaneous metastasis. ${ }^{1,2}$ It occurs more commonly between 50 to 70 years of age, usually in the first three years after diagnosis of the cancer. ${ }^{1}$

We described the case of a patient with florid lesions of $\mathrm{cu}^{-}$ taneous metastasis of breast cancer.

\section{CASE REPORT}

A 65-year-old woman had been developing plaques on her trunk over the past 6 months, lymph node enlargement on the left inguinal region (a painless, firm nodule) and loss of $15 \mathrm{~kg}$ during that time. She reported a previous history of breast cancer on the left breast 14 years prior, that was treated with radical mastectomy, and adjuvant chemotherapy (CT) and radiotherapy (RT). On dermatological examination, she had extensive erythematous-purplish firm plaques on both sides of the trunk (Figures 1 and 2). She underwent incision biopsy of a lesion on the right flank and was referred to the regional oncology service, with the clinical suspicion of breast cancer metastasis.

Histopathology from the biopsy sample revealed, in the reticular dermis, a neoplastic infiltrate of epithelial cells with pink cytoplasm, large nuclei, exuberant nucleoli and nuclear pleomorphism, with arrangement in masses and forming tubular structures in which lumen there was basophilic secretion (Figures 3, 4 and 5).

A specimen was sent to immunohistochemistry, with positive GATA-3 (breast transcription factor), estrogen receptor, progesterone receptor, cytokeratins (CKs) 40, 48, 50 and 50,6 kDa and

Received 29 March 2017.

Accepted 04 July 2017.

* Work conducted at the Dermatology Service, Hospital Universitário Cassiano Antônio Moraes, Universidade Federal do Espírito Santo (Hucam-Ufes), Vitória (ES), Brazil.

Financial support: None.

Conflict of interest: None.

Dermatology Service, Hospital Universitário Cassiano Antônio Moraes, Universidade Federal do Espírito Santo (Ufes), Vitória (ES), Brazil.

2 Empresa Brasileira de Serviços Hospitalares (EBSERH) and Dermatology Service, Hospital Universitário Cassiano Antônio Moraes, Universidade Federal do Espírito Santo (Ufes), Vitória (ES), Brazil.

3 Discipline of Internal Medicine/Dermatology, Universidade Federal do Espírito Santo (Ufes), Vitória (ES), Brazil.

4 Medical Residency Program in Dermatology, Universidade Federal do Espírito Santo (Ufes), Vitória (ES), Brazil.

MAILING ADDRESS:

Vítor Angelo Ferreira

E-mail: vitorangeloferreira@gmail.com

(C)2018 by Anais Brasileiros de Dermatologia 

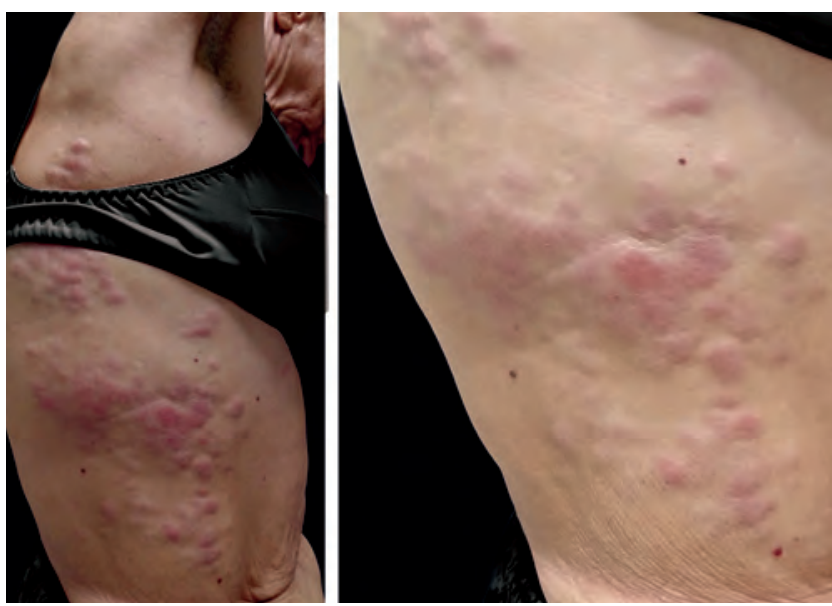

FIGURE 1: Firm and infiltrated red-purple plaques on the right side of the trunk

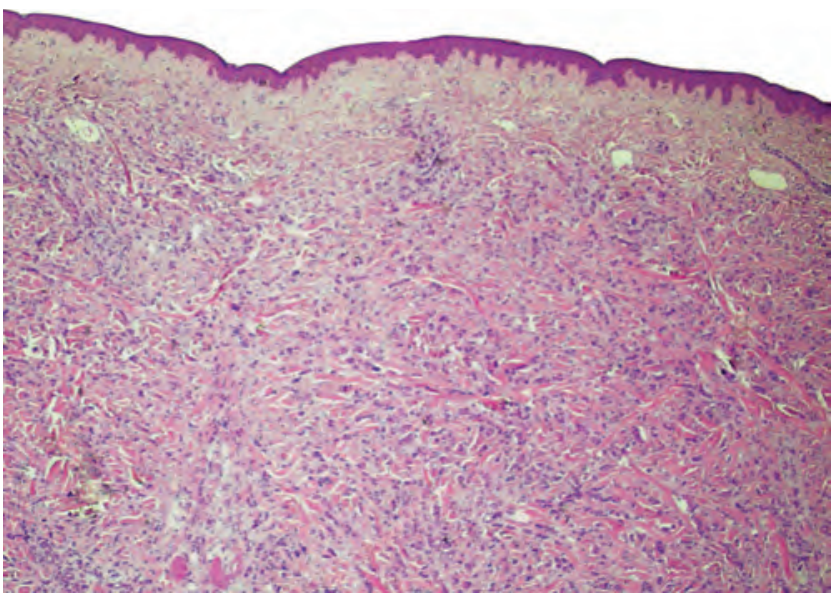

FiguRE 3: Preserved epidermis. In the reticular dermis we can observe the presence of an epithelial neoplasia with cells with pink cytoplasm and large nuclei organized in masses, resembling ducts (Hematoxylin \& eosin, X2,5)

HER-2 (2+). The expression of CKs confirmed the epithelial origin of the neoplastic cells and the expression of the estrogen receptor and GATA-3 favored the breast as the primary site of the carcinoma.

The patient received new cycles of CT e RT but died in one month and a half.

\section{DISCUSSION}

Cutaneous metastasis can be the first indication of recurrence of a cancer that was supposedly treated or it can represent the primary manifestation of an unknown internal malignancy. ${ }^{1}$

Cancer spread can occur through vascular or lymphatic routes, by contiguous growth or by iatrogenic implantation. ${ }^{3}$

The establishment of a metastasis depends on multiple cellular changes, such as loss of cell adhesion by the modification of intercellular adhesion proteins (cadherins) and adhesion between the cell and the extracellular matrix (integrins), favoring detachment and cellular migration; release of proteases (urokinase plasminogen activator, cathepsins and metalloproteinases), that promote extra-

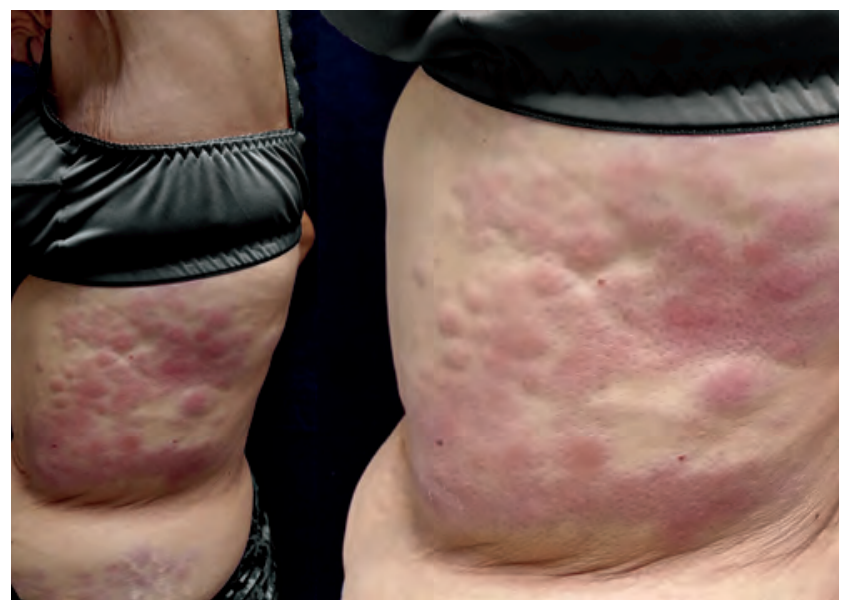

FIGURE 2: Firm and infiltrated red-purple plaques on the left side of the trunk. Note the "orange peel" aspect (carcinoma en cuirasse)

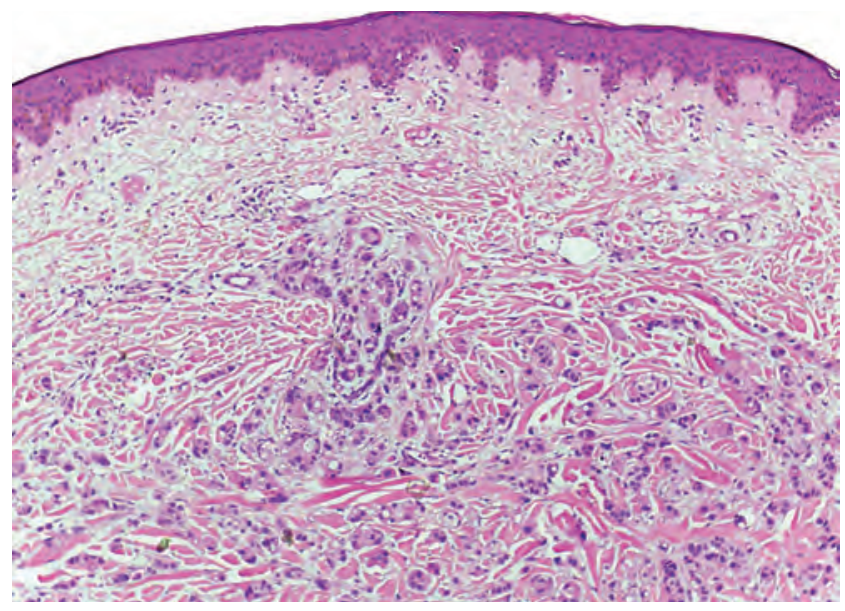

FIgURE 4: Atypical epithelial cells forming tubular structures, containing lumens - with basophilic secretion - and infiltrating the reticular dermis. We can also see the presence of occasional isolated neoplastic cells (Hematoxylin \& eosin, X10)

cellular matrix remodeling, favoring cell migration; automation, apoptosis and cell reproduction deregulation, leading to the multiplication of "immortal" neoplastic clones; and angiogenesis, with increase of the vascular endothelial growth factor (VEGF) and the human epidermal growth factor 2 (HER2) and decrease of the factor that inhibit it (angiostatin and endostatin), allowing for the nutritional supply of the tumor. ${ }^{4}$

The cutaneous metastasis from breast cancer begins as a non-adhered, painless erythematous papule on the chest (more frequently the anterior) and abdominal walls, that progresses to an inflammatory nodule measuring $1 \mathrm{~cm}$ to $3 \mathrm{~cm}$. It appears as a hardened, single or multiple lesion on the dermis or subcutaneous tissue. ${ }^{1,3}$ It can also present as an ulcer, erythematous macule or papule and appear on the opposite breast, in scars, arms, head and neck. ${ }^{2}$

According to Bolognia, Jorizzo and Rapini, cutaneous metastasis of a breast carcinoma can be clinically classified as: ${ }^{4}$

- Erysipeloid carcinoma: well-defined, raised erythematous plaque, resembling erysipelas. 


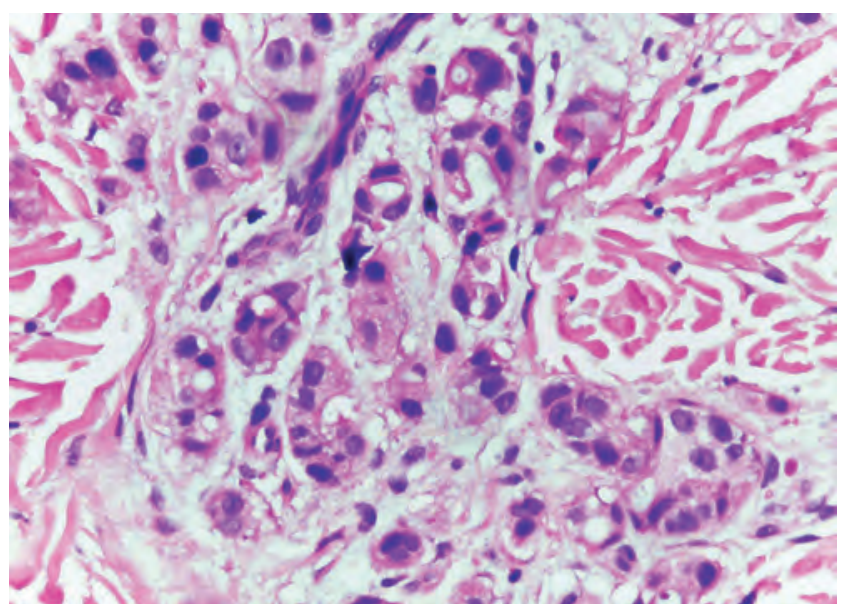

FIGURE 5: Epithelial cells with large nuclei, exuberant nucleoli, mild nuclear pleomorphism, resembling ducts with secretion in the lumen. The cells are interspersed with the reticular dermis (Hematoxylin \& eosin, X40)

- Telangiectatic carcinoma: erythematous papules with telangiectasia.

- Carcinoma en cuirasse: darkened lesion, translucent skin, with an "orange peel" aspect, resembling morphea.

- Neoplastic alopecia: cicatricial alopecia, anywhere on the scalp.

- Zosteriform metastasis: cutaneous nodules along a dermatome.

- Other possible presentations: sclerodermiform, inflammatory carcinoma and palpebral nodules. ${ }^{1}$

On microscopy, there are dermal deposits of pleomorphic, mitotic and neoplastic cells that can be seen in the vascular lumen. ${ }^{5}$
It also presents as an enlarged nodule in the deep dermis, formed by a solid aggregate of neoplastic cells, surrounded by fibrosis and minimal or absent inflammatory reaction. ${ }^{1}$

Most of the metastatic breast carcinomas express CK7 and CK19, estrogen and progesterone receptors, mammaglobin, GCDFP-15, CEA and E-cadherin but are negative for CK20, CK5/6, CD10 and TTF- 1,

The differential diagnoses are cysts, lipomas, adnexal tumors, dermatofibromas, keratoacanthomas, keloids, lymphocytomas, hansenomas, benign and malignant vascular lesions, morphea, erysipelas, radiation dermatitis, cicatricial alopecia, among others. ${ }^{3,4}$

Treatments are based on CT, RT, immunotherapy, surgical excision, heat and, many times, watchful waiting and palliation. ${ }^{4}$ Some medications used are imiquimod, with good results in localized lesions and trastuzumab (anti-HER2 antibody), in tumors with HER2 receptors. Patients with cutaneous metastasis of breast cancer without metastasis to internal organs can have a long survival or even be cured, according to reports of HER2-positive subtypes treated with trastuzumab. ${ }^{2}$

Metastatic cutaneous metastasis are manifestations of systemic recurrence; therefore, the therapy should never be only topical, requiring associated systemic CT even when only the skin is affected. ${ }^{2}$ The cutaneous response to the treatment can be used as a parameter in the evaluation of the systemic treatment. ${ }^{4}$

In general, the prognosis for cutaneous metastasis of breast cancer is poor, with a short survival.

According to the literature, the patient had the most frequent age group and site of lesions but, had extensive plaques on the chest instead of nodules, with the clinical aspect of carcinoma en cuirasse. Despite having received the recommended treatment, the poor prognosis was confirmed with the quick progression to death.]

\section{REFERENCES}

1. Campbell I, Friedman H, Alchorne M. Cutaneous metastasis of neoplasias: study carried out of 27 patients. An Bras Dermatol. 1995;70:409-18.

2. Cho J, Park Y, Lee JC, Jung WJ, Lee S. Case series of different onset of skin metastasis according to the breast cancer subtypes. Cancer Res Treat. 2014;46:194-9.

3. Bittencourt MJS, Carvalho AH, Nascimento BAM, Freitas LKM, Parijós AM. Cutaneous metastasis of a breast cancer diagnosed 13 years before. An Bras Dermatol. 2015;90:134-7.
4. Bolognia JL, Jorizzo JL, Rapini RP. Dermatology. 3rd ed. Mosby: Elsevier. 2012; Vol 2. 2049-55

5. Wong CY, Helm MA, Kalb RE, Helm TN, Zeitouni NC. The presentation, pathology and current management strategies of cutaneous metastasis. N Am J Med Sci. 2013;5:499-504

$\begin{array}{ll}\text { Vítor Angelo Ferreira } & \text { (ID) ORCID } 0000-0003-2672-5897 \\ \text { Karla Spelta } & \text { (ID) ORCID } 0000-0003-4790-443 X\end{array}$

\begin{tabular}{|c|c|}
\hline Lucia Martins Diniz & (iD) ORCID $0000-0001-8107-8878$ \\
\hline Elton Almeida Lucas & (iD) ORCID $0000-0002-6524-2030$ \\
\hline
\end{tabular}

How to cite this article: Ferreira VA, Spelta K, Diniz LM, Lucas EA. Exuberant case of cutaneous metastasis of breast cancer. An Bras Dermatol. 2018;93(3):429-31. 